\title{
Fairweather atmospheric electricity at Antarctica during local summer as observed from Indian station, Maitri
}

\author{
C Panneerselvam, C Selvaraj, K Jeeva, K U Nair, C P Anilkumar and S Gurubaran \\ Equatorial Geophysical Research Laboratory, Indian Institute of Geomagnetism, Krishnapuram, \\ Tirunelveli 627 011, India.
}

\begin{abstract}
Surface measurements of the atmospheric electrical parameters like Maxwell current, electric field and conductivity studied at the Indian station, Maitri $\left(70.75^{\circ} \mathrm{S}, 11.75^{\circ} \mathrm{E}, 117 \mathrm{~m}\right.$ above mean sea level), Antarctica, during austral summer have been analyzed for the years 2001 to 2004. A total of 69 days were selected which satisfied the 'fairweather' conditions, i.e., days with absence of high winds, drifting or falling snow, clouds, and fog effects. The diurnal variation curve of electric field and vertical current averaged for 69 fairweather days is a single periodic with a minimum at 03:00 UT and a maximum near 19:00 UT, which is very similar to the Carnegie curve. The correlation coefficient between these measured parameters has a high value (more than 0.9) for all the days. During fairweather days the measured current and field variations are similar and hence it is clear that the conductivity is more or less stable. During magnetically disturbed days, the dawn-dusk potential drop has clear influences on the diurnal variation and it modifies the conductivity. Apart from the day-to-day variation in low latitude thunderstorm activity, there are diurnal, seasonal, inter-annual variations in the electric potential and the currents, as well as solar influences on the measured parameters. This study will help us to examine the impact of solar and geophysical phenomena like solar flares, geomagnetic storms and substorms on the global electric circuit.
\end{abstract}

\section{Introduction}

Worldwide thunderstorm activity is believed to maintain the global electric potential between the ionosphere and the earth's surface (Wilson 1925). Tropical thunderstorms are considered as the main source for the electric field in the lower atmosphere, drawing current upward to the ionosphere. In this way, the global thunderstorm activity is able to maintain a time varying electric potential difference of $\sim 300 \mathrm{kV}$, directed downward between the equipotential surfaces of the ionosphere and the ground (Alderman and Williams 1996). The atmospheric vertical electric field, conductivity and total current density are the three closelyrelated parameters of atmospheric electricity that are required to obtain an adequate description of the fairweather atmospheric electric circuit (Israel
1973). The study of global electric circuit (GEC) can help in understanding the electrical environment of the earth's atmosphere. This approach can provide a good framework for exploring interconnections and coupling of various regions of the atmosphere. It can also provide information on the solar-terrestrial weather relationship and offers possibilities for exploring one of the traditional scientific problems, namely, that of associating changes in surface weather with the solar output (Herman and Goldberg 1978).

Thunderstorms are electrical generators whose global activity provides a current output that maintains a vertical potential difference between the ground and ionosphere. The GEC links the electric field and currents flowing in the lower atmosphere, the ionosphere and the magnetosphere. Measurements of atmospheric electrical

Keywords. Atmospheric sciences; climate; upper atmosphere; global electric circuit; thunderstorm; Antarctica; atmospheric electricity; fairweather; electric field. 
parameters will prove handy for any integrated approach involving all these regions. Long-term measurements would be considered useful for addressing some of the problems associated with global change. The measuring site needs to be free of atmospheric aerosols and convective activity, otherwise these would obscure the weak signatures representing the global thunderstorm activity (Israel 1973). Measurements from Antarctica also allow us to investigate meteorological effects, such as blizzards, wind turbulence, snowfall and clouds, on local electrical processes and enable us to understand the electrical climate of the Antarctic plateau (Cobb 1977).

The Antarctic station has been chosen as our instrument site for several reasons. Most importantly, the station is situated on the Antarctica plateau, a region where the atmosphere is more suited for making measurements of GEC (Park 1976; Cobb 1977). The global circuit involves lower atmosphere generators and upper atmosphere generators, the latter being significant over the polar caps. The Antarctic plateau supports a desert-like climate with clear skies and a very low atmospheric aerosol content. In summer, the prevailing winds are light, moving in a nearly constant direction and the atmosphere is relatively free of turbulent and convective motions (Byrne et al 1993). Thus, the wind induced conductivity fluctuations that contribute to meteorological noise in the measured atmospheric electric parameters are minimized when experiments are conducted in the Antarctic. The atmospheric electrical measurements studied in Antarctica are useful in investigating large-scale electrical processes that are unique at high latitudes (Byrne et al 1991). The downward airEarth current being delivered to the surface of the Antarctica plateau is larger than the global average owing to its location in high latitudes. Therefore, because of its orography, the Antarctica plateau is strongly coupled to the GEC. The ice surface is flat and is void of any obstructions and the electrical conductivity of the surface ice is several orders of magnitude higher than that of the air (Cobb 1977). Hence, the surface is essentially an infinite conducting plane at ground potential.

In this paper, we report observations of the atmospheric Maxwell current, electric field and polar conductivity made at Maitri for the years 2001 to 2004. The results have been compared with the earlier observations made in Antarctica. A variety of scientific experiments involving fluxgate magnetometer and proton precession magnetometer for detecting the earth's magnetic field variations and changes in the total geomagnetic field respectively, and riometer for D-region absorption studies, are being carried out by the Indian Institute of Geomagnetism, round the year.

\section{Description of the instrument}

Ground-based instruments that monitor atmospheric electrical parameters are widely used because they are inexpensive and can provide continuous long-term data. The most widely used ground-based sensors for the air-Earth current measurements are: Wilson plate (Israel 1973), the horizontal long-wire antenna (Ruhnke 1969), horizontal passive antenna (Harrison 1996) and spherical shell in the form of two hollow hemispheres (Burke and Few 1978). The horizontal long wire antenna, if placed in the atmosphere, will closely follow the electrical current variations of the atmosphere after the initial net charge on the antenna leaks off. When the antenna is shorted to the ground through a resistor, it will generate a voltage that is proportional to the air-Earth current. In the present experimental setup, a long wire of length $41.5 \mathrm{~m}$ and thickness $3 \mathrm{~mm}$ is kept horizontally stretched parallel to the ground at a height of $2 \mathrm{~m}$. The wire is mechanically supported by means of masts. By using teflon rods at their ends it is ensured that the antenna wire is electrically insulated from the supporting masts. The input is fed through the electrometer (Model AD 549) that has high input impedance and permits extremely low input bias current $\left(10^{-14} \mathrm{~A}\right)$. The electrometer measures the current up to $1 \mathrm{nA}$ (corresponding to the output voltages whose limit is $\pm 5 \mathrm{~V}$ ) with a feedback resistance of $5 \times 10^{9} \Omega$. A unity gain operational amplifier (LM308) amplifies the electrometer output signal. The amplified signal is then taken in a shielded cable over a distance of $\sim 20 \mathrm{~m}$ to the control room where it is fed to a PC-based data logger. The sensitivity of the digitized signal is $2.44 \mathrm{mV}$ that will correspond to a current of $\sim 0.5 \mathrm{pA}$. The data are recorded at a sampling interval of one second.

The atmospheric electric field has been measured with a vertical ac field mill made out of nonmagnetic stainless steel to reduce the contact potentials (Willett and Bailey 1983). The sensor plates and rotor in the field mill are of $8.5 \mathrm{~cm}$ diameter. The shaft of the ac motor $(220 \mathrm{~V}, 50 \mathrm{~Hz}, 1500 \mathrm{rpm})$ is grounded with a carbon brush. The signal from the field mill is amplified with Op-Amp 1596 which is mounted inside the field mill. Further details are available in Deshpande and Kamra (2001).

The electrical conductivity of both polarities has been measured with Gerdien's apparatus. It consists of two identical cylindrical condensers of stainless steel joined with a $U$ tube and has a common fan to suck the air through the two tubes. The critical mobility of each condenser is adjusted to be $3.60 \times 10^{-4} \mathrm{~m}^{2} \mathrm{~V}^{-1} \mathrm{~s}^{-1}$. The signal from each condenser is amplified separately with Analog Devices $311 \mathrm{~K}$, and the box containing the circuitry is fitted 
on the tube. The time constant of each tube is 5 seconds. Gerdien's apparatus is vertically installed at Maitri such that the inlets of the tubes are about $24 \mathrm{~cm}$ above the ground. The fan for air suction is covered with a conical cap to avoid accumulation of snow or rain. Care was taken to use military grade components in all electronic circuits which maintain their characteristics in subzero temperatures. The amplified signals from both field mill and Gerdien's apparatus are fed through tefloninsulated coaxial cables to a PC-based data acquisition system, which is kept inside a hut that is $4 \mathrm{~m}$ in height and $50 \mathrm{~m}$ away from the field mill. The equipment is operated at a sampling interval of one second. The maintenance of the instruments, especially cleaning of their insulators, was done daily. The zero shifts are checked twice a day and are corrected if the deviations are appreciable (Deshpande and Kamra 2001).

The sources other than the air-Earth conduction current that contribute to the measured current density are (i) the convection current, (ii) the displacement current, (iii) the point discharge current, (iv) the precipitation current, and (v) the lightning current (Israel 1973). The sum of all these currents is collectively called the Maxwell current. Out of these, the convection current arises when the charge carriers are moved by the winds, and hence it is a possible source for the locally induced component in the measured current. The lightning and precipitation currents are not considered since the datasets selected are during fairweather periods. The location of the experimental setup on a barren land does not favour point discharge current because the sharp edges provided by plants and trees allow for point-discharge currents but in Antarctica there are no trees and plants hence, contribution of point-discharge current is negligible. The displacement current can be minimized by choosing an appropriate $\mathrm{RC}$ time constant in the detection circuit. The effective area of the present experimental setup is $66.1 \mathrm{~m}^{2}$ calculated from the formula $S=h c / \varepsilon$ (Kasemir and Ruhnke 1959; Tammet et al 1996), $\varepsilon$ being the dielectric constant of air, $c$ the capacity of the antenna and $h$ the height of the antenna above the ground. Here the value for $c=298.2 \mathrm{pF} ; h=2 \mathrm{~m}$. The total current density can be estimated by dividing the measured current by the effective area of the antenna.

\section{Site description}

The Indian Antarctic station, Maitri is located in the Schirmacher oasis in the Dronning Maud Land, East Antarctica (117 $\mathrm{m}$ above the mean sea level). Antarctica has only around $2 \%$ of its area that is free from ice. The nearest steep cliff of the eastwest trending glacier on the southern side of the station is more than $700 \mathrm{~m}$ away from the station and is $300 \mathrm{~m}$ in height. The snow-covered surface during summer season was more than half a kilometer away from the station. The instruments were installed on barren land near the station. The surface of the station area is mainly covered by sandy and loamy sand types of soil. The solar zenith angle at Maitri varies from $48^{\circ}$ to $88^{\circ}$ during summer months. There was no sunset till the third week of January, but periods of short nights slowly increased during February. The variations in surface meteorological parameters like wind speed, wind direction, snowfall, pressure, visibility and all other parameters are systematically monitored by the India Meteorological Department. The cloud cover over the station occurs mainly under the influence of subpolar low-pressure systems and shows an alternating sequence of the sky changing from overcast to clear as the system moves away (Deshpande and Kamra 2001).

\section{Data selection and analysis}

The selection of the fairweather days has been made with the standard procedure adopted in the past (Reiter 1985). In the present study two types of datasets were looked at for analysis. For one type of study, a set of days during which fairweather conditions prevailed all through the day were considered, and the hourly averages were computed to yield the diurnal variation (Troshichev et al 2004). Features corresponding to the UT variation were then looked for. If thunderstorm activity was responsible for the generation of global electric circuit and its variation with time, one would expect in the average pattern a maximum in the measured electrical parameters near 19:00 UT and a minimum near 03:00 UT. The other type of study included fairweather days on which different geomagnetic conditions like magnetic quiet days, moderate and disturbed days (geomagnetic storm days) were considered.

Geomagnetic storm is defined in general, by the existence of a main phase during which the magnetic field at earth's surface is depressed and this depression is caused by a westward ring current in the magnetosphere. The main phase of geomagnetic storm is characterized by the 'frequent' occurrence of intense substorms. It is believed that the magnetospheric substorm results when the energy stored in the magneto-tail is explosively released towards the inner magnetosphere and finally deposited as heat energy in the upper atmosphere. During substorm activity some of the ionized particles drift around the Earth and 
enhance the ring current as well as energy. Field aligned current intensity and particle precipitation are increased. Dramatic increases are observed in ionospheric current which flows across the night side auroral ionosphere. The brightening and expansion of the auroral forms are observed when viewed from the ground. The electric field due to solarwind/magnetosphere dynamo map down to auroral ionosphere and produced potential difference across the polar cap of the order of tens of kilovolts depending on the geomagnetic conditions. It will also have measurable effects on the vertical electric field and current. The auroral or magnetic substorm activity lasts from about one hour to a few hours. Such studies help in the investigation of the influence of high latitude upper atmospheric contribution to the measured electrical parameters. To separate the thunderstorm, ionospheric/magnetospheric contribution we have carried out the principal component analysis (Gnanadesikan 1977) for the electrical parameters.

The most widely used multivariate method, is the principal component analysis (PCA), which is a classical statistical method. PCA is a transformation, which transforms a number of (possibly) correlated variables into a (smaller) number of uncorrelated variables called 'principal components' (PCs). The objectives of this analysis are to reduce the dimensionality of the dataset and to identify new meaningful underlying variables. The basic idea of principal component analysis is to describe the dispersion of an array of $n$ points in $p$-dimensional space by introducing a new set of orthogonal linear coordinates so that the sample variances of given points with respect to these derived coordinates are in decreasing order of magnitude. The mathematical technique used in PCA is called eigen analysis: we solve for the eigenvalues and eigenvectors of a square symmetric matrix of covariance. The eigenvector associated with the largest eigenvalue has the same direction as the first principal component and contains the most variance in the data. The eigenvector associated with the second largest eigenvalue determines the direction of the second principal component and contains the most variance when the first component is removed from the data. The sum of the eigenvalues equals the trace of the square matrix and the maximum number of eigenvectors equals the number of rows (or columns) of this matrix. A common way to find the principal components of a dataset is by calculating the eigenvectors of the data correlation matrix. These vectors give the directions in which the data cloud is stretched most. The projections of the data on the eigenvectors are the principal components. The corresponding eigenvalues give an indication of the amount of information represented by the respective principal

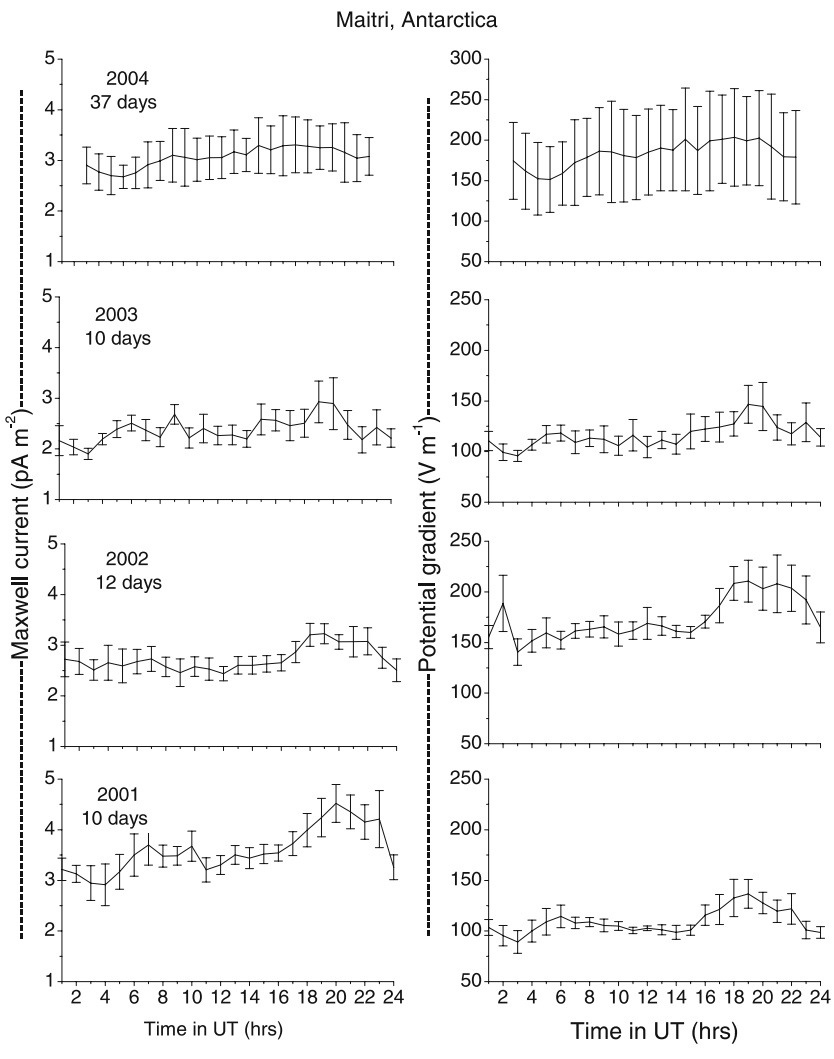

Figure 1. The diurnal variation of electric field and Maxwell current density averaged for the 69 'fairweather' days for the years 2001 to 2004 .

components. Principal components corresponding to large eigenvalues, represent much information in the dataset and thus tell us much about the relations between the data points. This technique of analysis has been used to study the day-to-day variability of the measured atmospheric electrical parameters in the present investigation.

\section{Results and discussion}

The diurnal variation of the hourly averaged values of the geoelectric field and Maxwell current density averaged for the 69 'fairweather' days for the years 2001 to 2004 is depicted in figure 1. We define a fairweather day when there is no snowfall at the measuring site, high clouds less than 3 octas throughout the day, and wind speed less than or equal to $10 \mathrm{~m} \mathrm{~s}^{-1}$. The diurnal variation has a single periodic feature with a minimum at 03:00 UT and a maximum at 19:00 UT. This diurnal trend is consistent with the familiar 'Carnegie curve' variation. This variation has been widely observed, and according to classical theory, it is generally attributed to the variation with time of day of the number of thunderstorms across the globe (Roble 1985). Part of the day-to-day variations may be ascribed to the solar wind/magnetospheric 


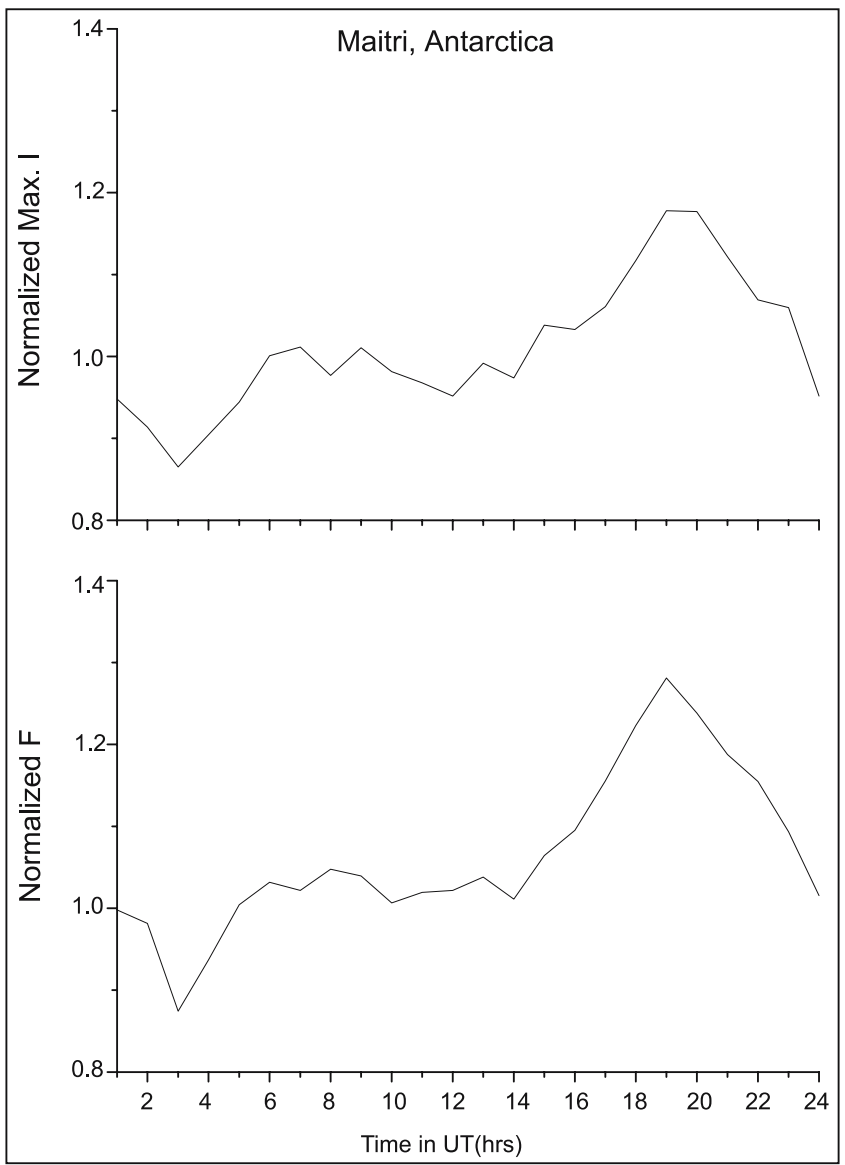

Figure 2. Normalized diurnal variations of Maxwell current density and electric field averaged for 69 fairweather days at Maitri.

and ionospheric contribution because our station is outside the region of auroral oval during the magnetically quiet times and is encompassed by the auroral oval under magnetically disturbed conditions. According to the modern GEC theory one has to include the magnetospheric/ionospheric contributions also. Electric fields are generated in the ionosphere by the dynamo action and by the interaction of the magnetosphere with the solar wind and its frozen-in interplanetary magnetic field (IMF). This part corresponds to mainly horizontal potentials of the order of tens of kilovolts. This horizontal potential will have effect on the measured atmospheric electrical parameters. Through the observations made during the sunspot maximum year, one would expect more magnetic storms that may produce large day-to-day variation in electric field and Maxwell current density.

In this presentation we normalize our observations by the corresponding daily mean value, in which much of the variations on day-to-day timescale has been removed. The low-latitude thunderstorm generators, the local atmospheric conductivity, and the cosmic ray flux all have variations extending through this timescale. We

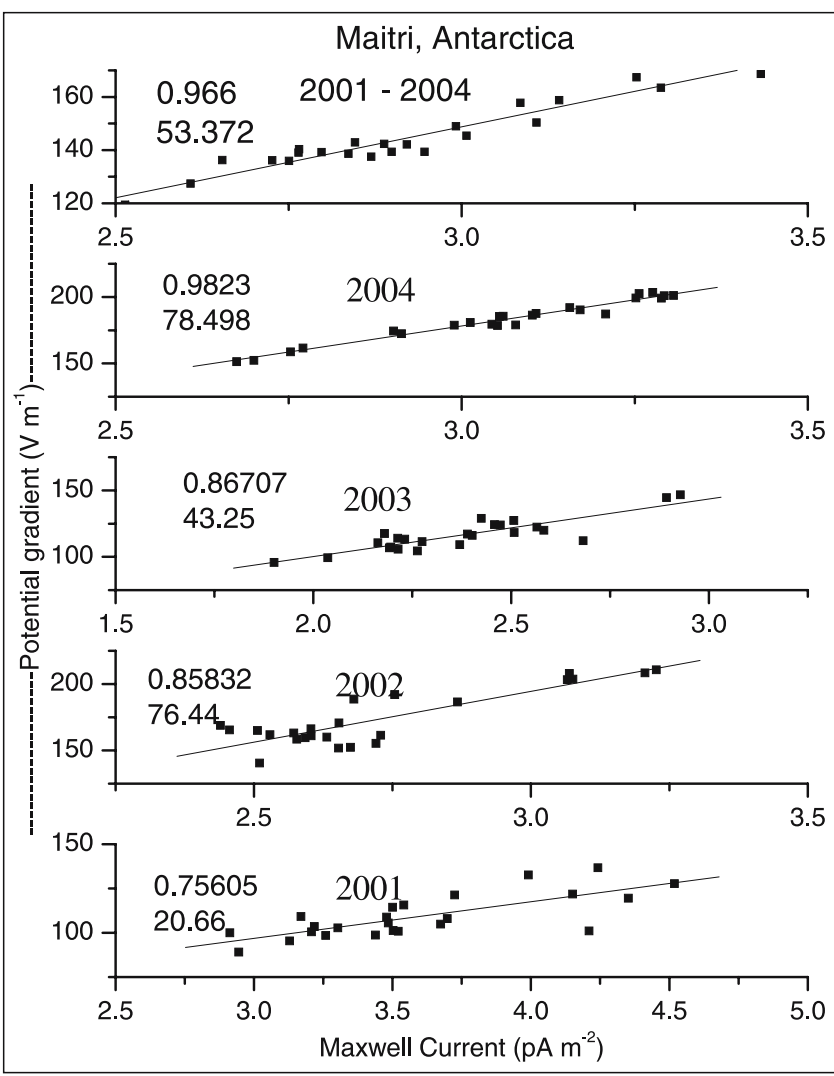

Figure 3. Maxwell current density plotted against the electric field variations for 69 fairweather days.

can then replot the experimental atmospheric electricity data as daily fairweather 'Carnegie-like' curves related to the diurnal variation of the global thunderstorm activity (Reiter 1992). Figure 2 shows diurnal variation of normalized Maxwell current and electric field for all 69 selected fairweather days for the years 2001 to 2004. Normalization of the experimental data reduces their dispersion, revealing a regular change of measured parameters as a function of universal time.

The Maxwell current density plotted against the electric field variations are shown year-wise and in average in figure 3. The correlation coefficient for these measured parameters has a high value (more than 0.9) during the selected fairweather days averaged for four years. From the slope of the curve the conductivity was calculated and its value is $2.0 \times 10^{-14} \mathrm{~S} / \mathrm{m}$. The upper left corner in each panel values are correlation coefficient (upper) and slope (lower). With the measured atmospheric electrical parameters, the calculated Maxwell current density (calculated form Ohms law $\mathrm{J}=\sigma \mathrm{E}$ ) was compared with measured Maxwell current density. Figure 4 shows the atmospheric electrical conductivity measurements during the fairweather conditions. These measured conductivity is more or less constant during the fairweather conditions. 


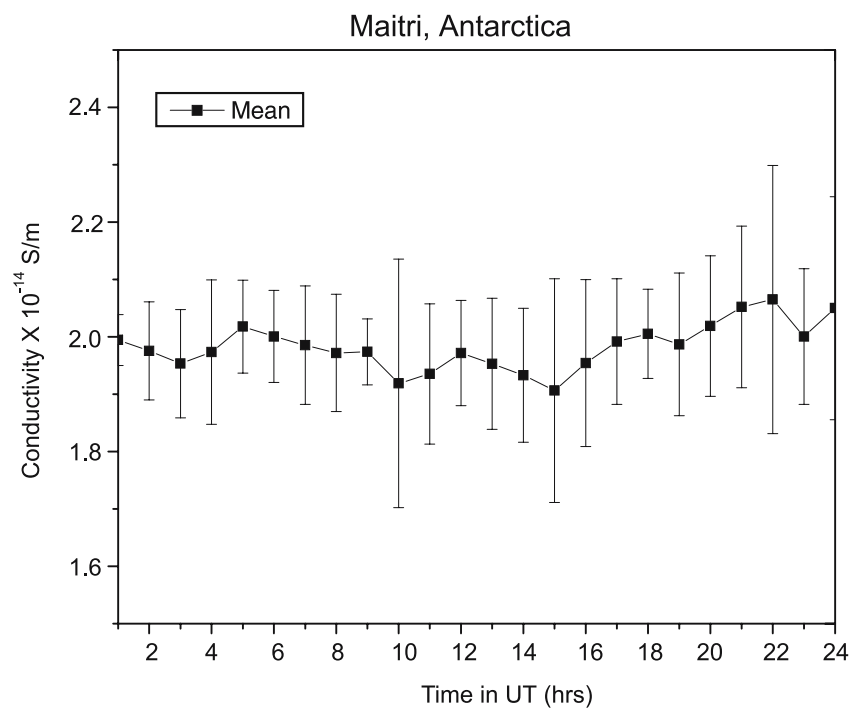

Figure 4. Diurnal variation of electrical conductivity averaged for 28 fairweather days.

An attempt has been made to identify the geomagnetic influences on the Maxwell current density and electric field associated with dawn-dusk potential difference across the polar cap. The dawn-to-dusk horizontal potential difference (up to $\sim 100 \mathrm{kV}$ ) across the polar cap arising from the interaction of the solar wind with the magnetosphere, which causes the two-cell convection pattern in the polar region, one side it is clockwise and the other is anti-clockwise. This enhanced clockwise cell would map to increased upward vertical electric field and current at the ground. The anti-clockwise cell dawn convection would map to decrease the atmospheric vertical electric field and current. Our data (69 fairweather days) were classified into three sets according to the geomagnetic conditions: geomagnetically quiet, moderately disturbed and disturbed days. The international quiet and disturbed days are downloaded from the website "World Data Center for Geomagnetism, Kyoto". The selection of the quietest days (Q-days) and most disturbed days (D-days) of each month is deduced from the $K_{p}$ indices on the basis of three criteria for each day: Geomagnetic disturbances can be monitored by ground-based magnetic observatories recording the three magnetic field components. The global $K_{p}$ index is obtained as the mean value of the disturbance levels in the two horizontal field components, observed at 13 selected stations.

The sum of the eight $K_{p}$ values.

1. The sum of squares of the eight $K_{p}$ values.

2. The maximum of the eight $K_{p}$ values.

According to each of these criteria, a relative order number is assigned to each day of the month, the three order numbers are averaged and the days with the lowest and the highest mean order

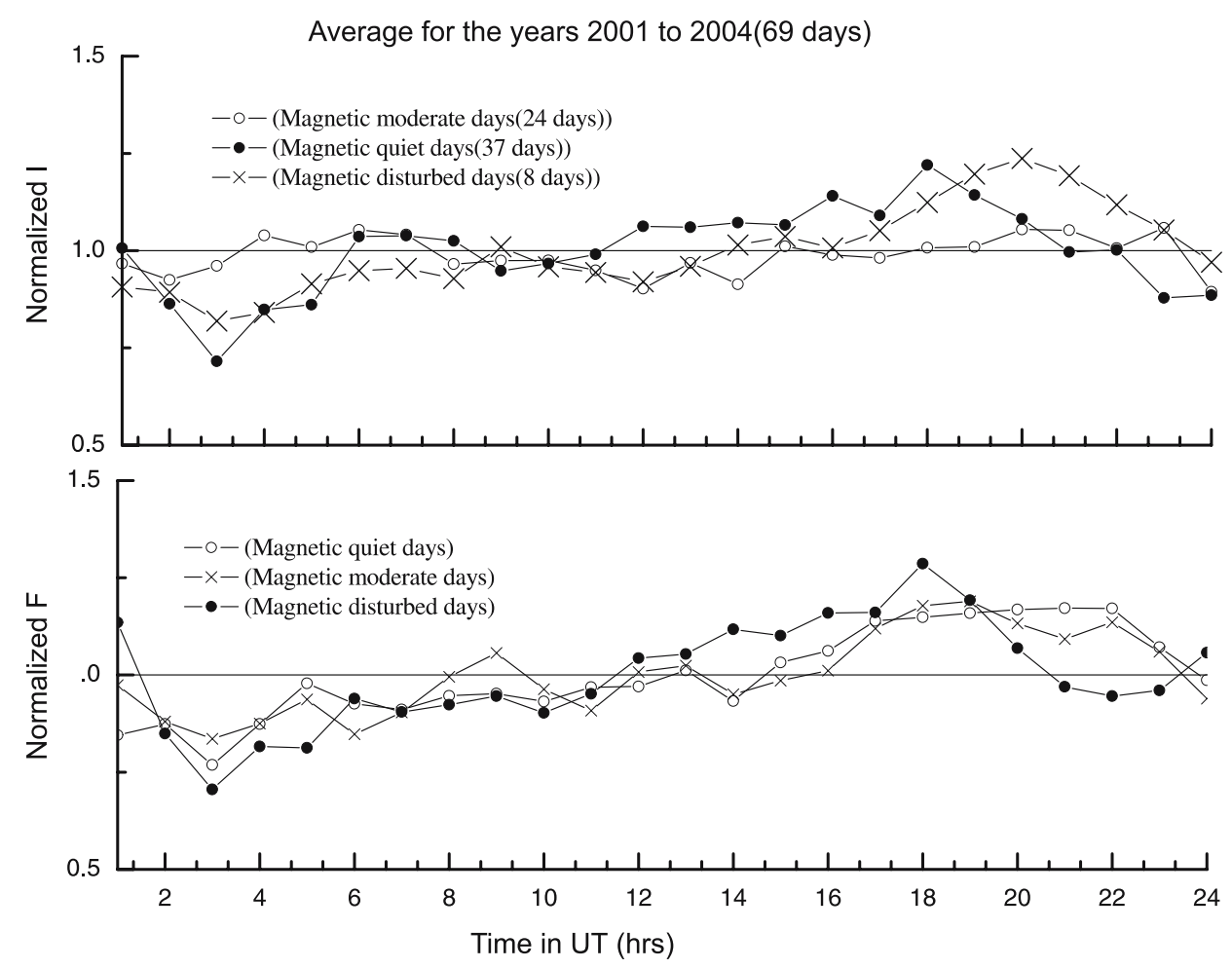

Figure 5. Normalized electric field and Maxwell current density during different geomagnetic conditions. 
(a)
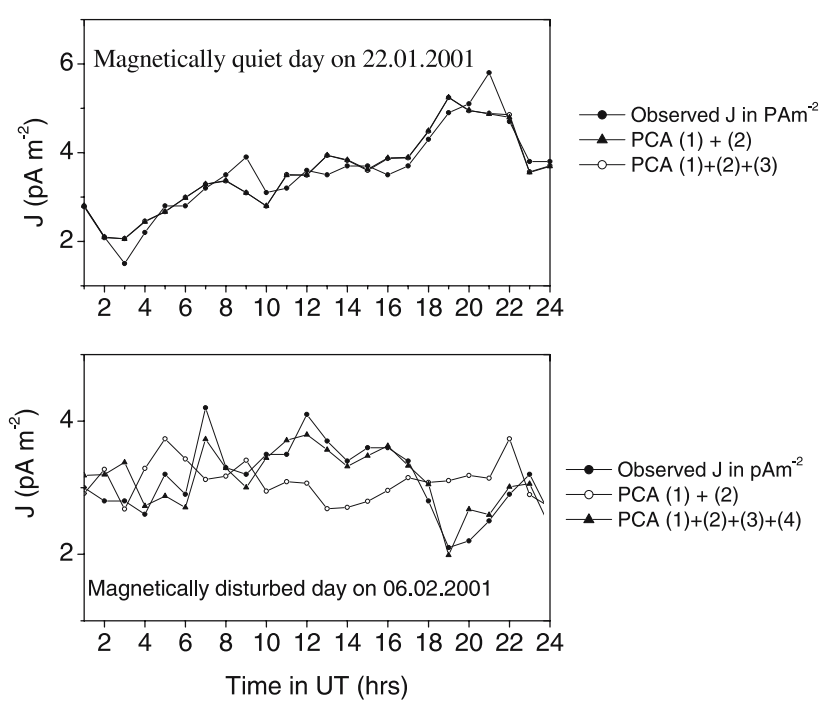

(b)
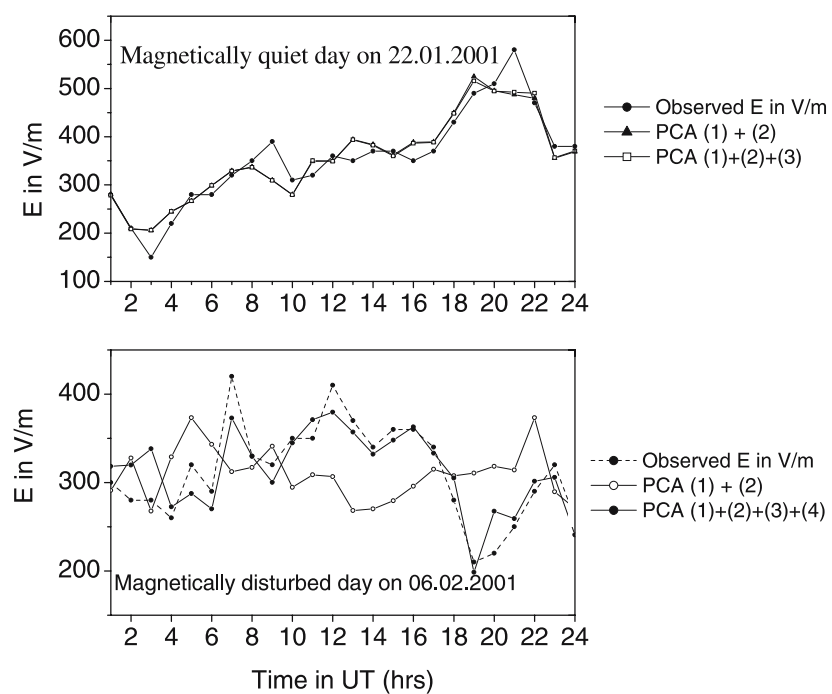

Figure 6. Diurnal variation of (a) Maxwell current and (b) electric field during magnetic quiet and disturbed days using PCA.

numbers are selected as the five (respectively ten) quietest and the five most disturbed days. The normalized Maxwell current density and electric field diurnal variations for magnetically quiet, moderate and disturbed days are shown in figure 5 . We had 37 quiet days, 24 moderately disturbed days and 8 disturbed days among the selected 69 fairweather days. During magnetically quiet and moderately disturbed days the variation of the Maxwell current density and electric field follows the global thunderstorm variation like the famous 'Carnegie curve'. During magnetically disturbed days the measured electrical parameters show greater variation deviated with a modification upon the UT pattern. Our station is under the influence of auroral oval during the geomagnetic disturbed period (Rajaram et al 2001). During magnetically quiet days the variation of atmospheric electrical parameters follows the famous 'Carnegie curve' a minimum at 03:00 UT and a maximum at 19:00 UT. During moderately disturbed days, the UT pattern is modified and follows the maximum at about 19:00 UT but during disturbed days the diurnal pattern is modified due to the magnetospheric/ionospheric contribution to the measured parameters. To separate the thunderstorm, ionospheric/magnetospheric contribution we have used the Principal Component Analysis (PCA) for the electric field and Maxwell current density. The first component gives information about the thunderstorm contribution to the global electric circuit. The second and higher components will give us information on the upper atmospheric contribution namely from the ionosphere and the magnetosphere. During magnetic quiet days the first order component (thunderstorm contribution) contributes the maximum. The remaining component's (second and higher) contribution is very less. The measured electrical parameter and the PCA first order component was compared and found identical. Geomagnetically disturbed days were also used for the PCA analysis and the observed diurnal pattern compared with the first order component. We found that there is a deviation from the measured atmospheric electrical parameters. Then we have added the second and third order component with first order, the PCA first order with the second and third order components compared with the observed diurnal curve and we found it is identical. From the PCA it is clearly seen that during magnetically disturbed days the ionospheric/magnetospheric contribution modifies the diurnal pattern of the measured atmospheric electrical parameters, which is shown in figure 6(a) and $6(\mathrm{~b})$.

\section{Conclusions}

During magnetically quiet and moderate conditions, the variations of measured atmospheric electrical parameters are similar to the famous fairweather 'Carnegie curve'. But, during geomagnetically disturbed conditions the diurnal pattern is different from the Carnegie curve and is modified by the ionospheric/magnetospheric contributions. With the continuous measurements of atmospheric electrical parameters and geomagnetic field variations, there is scope for addressing the problems related to the modulation of GEC by the influence of magnetosphere-ionosphere coupling processes on the near-surface electrical parameters in the polar caps. 


\section{Acknowledgements}

The authors gratefully acknowledge Dr Pallamraju and another referee for their valuable remarks and corrections, which helped in making improvements on the original version. The instruments field mill and conductivity were provided by the Indian Institute of Tropical Meteorology, Pune, under a collaborative study. We are also grateful for the support by the Department of Science and Technology (DST), Government of India, and the logistic support provided by the Department of Ocean Development, Government of India, for conducting a variety of experiments at the Indian Antarctic station, Maitri.

\section{References}

Adlerman E J and Williams E R 1996 Seasonal variation of the global electric circuit; J. Geophys. Res. 101 $29,679-29,688$.

Burke H K and Few A A 1978 Direct measurements of the atmospheric conduction current; J. Geophys. Res. $\mathbf{8 3}$ 3093-3098.

Byrne G J, Benbrook J R and Bering E A 1991 Balloon observations of atmospheric electricity above the south pole: vertical electric field, conductivity and conduction current; J. Atmos. Terr. Phys. 53(9) 859-868.

Byrne G J, Benbrook J R, Bering E A, Few A A, Morris G A, Trabucco W J and Paschal E W 1993 Ground based instrumentation for measurements of atmospheric conduction current and electric field at south pole; J. Geophys. Res. 98 2611-2618.

Cobb W E 1977 Electrical Process in Atmospheres (eds) Dolezalek H and Reiter R (Darmstadt: Steinkopff) 161-167.

Deshpande C G and Kamra A K 2001 Diurnal variations of the atmospheric electric field and conductivity at Maitri, Antarctica; J. Geophys. Res. 106 14,207-14,218.

Gnanadesikan R 1977 Methods for statistical data analysis of multivariate observations; John Wiley and Sons, New York.
Harrison R G 1996 An atmospheric electrical voltmeter follower; Rev. Sci. Instrum. 67(7) 2636-2638.

Herman J R and Goldberg R A 1978 Sun, Weather and Climate; Scientific and Technical Information Branch, National Aeronautics and Space Administration, Washington, D.C.

Israel H 1973 Atmospheric Electricity; vol. II, Isr. Program for Sci. Transl., Jerusalem.

Kasemir H W and Ruhnke L H 1959 Antenna problems of measurement of the air-Earth current; In: Recent Advances in Atmospheric Electricity (ed.) Smith L G (New York: Pergamon) pp. 137-147.

Park C G 1976 Solar magnetic sector effects on the vertical atmospheric electric field at Vostok, Antarctica; Geophys. Res. Lett. 3(8) 475-478.

Rajaram G, Dhar A and Kumar S 2001 Response of geomagnetic variations and $30 \mathrm{MHz}$ riometer absorption at Indian Antarctic station Maitri, to conditions of 'Zero' and 'high' solar wind; Adv. Space Rev. 28(11) $1661-1667$.

Reiter R 1985 Fields, Currents and Aerosols in the Lower Troposphere; published for the division of Atmospheric Sciences, National Science Foundation, Washington, D.C., by Amerind Publishing Co. Pvt. Ltd., New Delhi.

Reiter R 1992 Phenomena in Atmospheric and Environmental Electricity (New York: Elsevier) 541.

Roble R G 1985 On solar-terrestrial relationships in atmospheric electricity; J. Geophys. Res. 90 6000-6012.

Ruhnke L H 1969 Area averaging of atmospheric electric currents; J. Geomagn. Geoelectr. 21 453-462.

Tammet H, Israelsson S, Knudsen E and Tuomi T J 1996 Effective area of a horizontal long-wire antenna collecting the atmospheric electric vertical current; J. Geophys. Res. 101 29,671-29,677.

Troshichev O A, Frank-Kamenetsky A, Burns G, Fuellekrug M, Rodger A and Morozov V 2004 The relationship between variations of the atmospheric electric field in the southern polar region and thunderstorm activity; Adv. Space Res. 34(8) 1801-1805.

Willett J C and Bailey J C 1983 Contact-potential and surface charge effects in atmospheric-electrical instrumentation, NRL, Memo. Rep. 5063, Nov. Res. Lab. Washington, D.C.

Wilson C T R 1925 The electric field of the thundercloud and some of its effects; Proc. Phys. Soc. London, Sect. A $11132 \mathrm{D}$ 